\title{
Influence of Elastic Anisotropy on Structural Nanoscale Textures
}

\author{
Pol Lloveras, ${ }^{1,2}$ Teresa Castán, ${ }^{1,2}$ Marcel Porta, ${ }^{1,2}$ Antoni Planes, ${ }^{1,2}$ and Avadh Saxena ${ }^{2,3}$ \\ ${ }^{1}$ Departament d'Estructura i Constituents de la Matèria, Universitat de Barcelona, Diagonal 647, 08028 Barcelona, Catalonia, Spain \\ ${ }^{2}$ Institut de Nanociència i Nanotecnologia de la Universitat de Barcelona, Barcelona, Spain \\ ${ }^{3}$ Theoretical Division, Los Alamos National Laboratory, Los Alamos, New Mexico 87545, USA
}

(Received 21 February 2008; published 25 April 2008)

\begin{abstract}
We study the influence of elastic anisotropy on nanoscale precursor textures that exist in some shapememory alloys and show that tweed occurs in the limit of high elastic anisotropy while a nanocluster phase-separated state occurs for values of anisotropy inhibiting the formation of martensite. These results are consistent with specific heat data, elastic constant measurements, and zero-field cooling or field cooling experiments in nonstoichiometric NiTi alloys.
\end{abstract}

DOI: 10.1103/PhysRevLett.100.165707

PACS numbers: 64.70.K-, 62.20.D-, 81.30.Kf

Systems exhibiting spatially inhomogeneous states are now a subject of increasing interest [1,2]. Prototypical examples are tweed textures observed in some martensitic materials as precursors [3] to the structural transition. Tweed [4] refers to the contrast anomaly observed in TEM consisting of striations parallel to the traces of $\{110\}$ planes appearing at intervals of few nanometers [5]. It originates from a static, long length scale elastic modulation of a very small strain amplitude that arises as the natural cooperative response of anisotropic long-range (elastic) interactions to local inhomogeneities [6] coupling to strain. Nanoscale precursor modulations are not exclusive to martensitic or ferroelastic systems, but instead they occur in a broad class of ferroic materials including ferromagnetic $[7,8]$ and ferroelectric materials [9]. The specific symmetry properties of the anisotropy determine the selected pattern.

In cubic shape-memory alloys (SMA) the possibility of tweed is enhanced by the existence of easy shear directions along $\{110\}$ planes - reflected in a low value of shear modulus $C^{\prime}$ - which define natural channels that propagate correlations. This is an essential feature since it circumvents the expected cutoff of correlations associated with disorder [10]. Thus, one expects that reducing the elastic anisotropy factor $(\mathcal{A})$, defined as the ratio between the two relevant shear moduli $\mathcal{A}=C_{44} / C^{\prime}$, will inhibit the occurrence of tweed. In the case of SMA, tweed has been observed only in materials with a significant value of $\mathcal{A}$. Examples are [11] $\mathrm{Ni}-\mathrm{Al}, \mathrm{Cu}-\mathrm{Zn}-\mathrm{Al}$ (and other $\mathrm{Cu}-$ based alloys), and $\mathrm{Fe}-\mathrm{Pd}$, among others. A completely different case is that of Ni-Ti which exhibits a very rich pretransitional behavior [12] but no signature of tweed striations. The diffuse TEM contrast reported in iron-doped Ni-Ti $[\mathrm{Ni}-\mathrm{Ti}(\mathrm{Fe})]$ has been found to correspond to very small domains of the incoming phase of almost spherical shape [13]. Energy-filtering dark-field imaging techniques enable one to visualize tiny domains down to $5 \mathrm{~nm}$ which are not elongated in specific directions [13]. This morphology is definitely different from that responsible for the striations in the tweed contrast $[14,15]$. In this case, upon cooling, the interacting domains line up and enhance the crosshatched directionality of the tweed correlations.

Murakami et al. [13] have suggested that the peculiar behavior of Ni-Ti might be due to its low value of $\mathcal{A}$. Nevertheless, the role of elastic anisotropy on ferroelastic (martensitic) transitions, and specifically on the resultant microstructure, has not been explored previously. Here we use an appropriate model and systematically study the role of $\mathcal{A}$. We find that there is a crossover from twinned martensite to a nanocluster phase-separated state (i.e., no twinning and no long-range order) as the anisotropy $\mathcal{A}$ is reduced below a certain value. This picture is corroborated by the behavior of relevant response functions: (i) The heat capacity exhibits a peak that disappears at low values of $\mathcal{A}$ consistent with experiments [16] on $\mathrm{Ni}-\mathrm{Ti}(\mathrm{Fe})$. (ii) The elastic constant $C^{\prime}$ shows a qualitatively different temperature behavior at high and low anisotropy in agreement with experiments [17] on Ni-rich $\mathrm{Ni}$-Ti. (iii) We have also obtained a different elastic behavior in the field cooling (FC) (stress) versus zero-field cooling (ZFC) (no stress) simulations in excellent agreement with recent experimental data reported in [18]. All these results suggest that the nanocluster phase-separated state found at low temperatures and at low values of $\mathcal{A}$ shares several features with structural glasses. Note that similar ideas may be applicable to the high-temperature superconductors and colossal magnetoresistance materials where nanoscale phaseseparated states (albeit of other degrees of freedom such as charge or orbital ordering) have been discussed in the literature [1,2].

We consider a 2D Ginzburg-Landau free energy for square-to-rectangular structural transitions which includes local coupling to disorder [19-21]. The symmetry adapted strains [22] $e_{1}, e_{2}$, and $e_{3}$ stand, respectively, for hydrostatic, deviatoric, and shear modes. Here, $e_{2}$ is the order parameter (OP) while $e_{1}$ and $e_{3}$ are (secondary) non-OP strains. The three strain components are not independent because they are obtained as derivatives of the same under- 
lying displacement field. The free-energy density is written as the summation of three contributions: $f\left(e_{1}, e_{2}, e_{3}\right)=$ $f_{h}\left(e_{2}\right)+f_{\text {grad }}\left(\vec{\nabla} e_{2}\right)+f_{\text {non-OP }}\left(e_{1}, e_{3}\right)$. The first is the homogeneous Landau part accounting for the required nonlinearities in the $\mathrm{OP}$ and for local coupling to inhomogeneities through the harmonic coefficient. The spatial inhomogeneities are described by a static perturbing field, corresponding to quenched-in disorder, for instance, statistical compositional fluctuations, always present in alloys. Here $f_{\text {grad }}$ is the Ginzburg (gradient) term responsible for the interface energy in the OP. Finally, $f_{\text {non-OP }}$ is the contribution due to the non-OP components of the strain that we assume to be harmonic. Namely, $f_{h}\left(e_{2}\right)=$ $\frac{1}{2} A_{2}[T, \eta(\vec{r})] e_{2}^{2}+\frac{1}{4} \beta e_{2}^{4}+\frac{1}{6} \gamma e_{2}^{6}, \quad f_{\text {grad }}\left(e_{2}\right)=\frac{1}{2} \kappa\left|\vec{\nabla} e_{2}\right|^{2}$, and $f_{\text {non-OP }}\left(e_{1}, e_{3}\right)=\frac{1}{2} A_{1} e_{1}^{2}+\frac{1}{2} A_{3} e_{3}^{2}$. Here $A_{1}=\left(C_{11}+\right.$ $\left.C_{12}\right)$ is the bulk modulus and $A_{2}=\left(C_{11}-C_{12}\right)=2 C^{\prime}$ and $A_{3}=4 C_{44}$ are elastic constants associated, respectively, with deviatoric and shear modes. $A_{2}=2 C^{\prime}$ is expected to partially soften with temperature. Moreover it depends on the disorder through $A_{2}[T, \eta(\vec{r})]=\alpha_{T}\left(T-T_{c}\right)+\alpha_{\eta} \eta(\vec{r})$, where $T_{c}$ is the lower stability limit of the hightemperature phase in the clean limit and $\eta(\vec{r})$ is a random variable Gaussian distributed around zero and with spatial correlations described by an exponential pair correlation function. Note that this kind of coupling has the effect of producing a distribution of local transition temperatures. Here $\beta$ and $\gamma$ are elastic nonlinearity parameters.

The strain tensor components must satisfy compatibility constraints in order to ensure the lattice integrity. Formally they are expressed through the Saint-Vénant equation that links the different components of the (symmetric) strain tensor [21]. After minimization of the total free energy with respect to $e_{1}$ and $e_{3}$ and taking into account the compatibility condition [21] one obtains that $f_{\text {non-OP }}$ has the form $f_{\text {non-OP }}=\int e_{2}(\vec{r}) U\left(\vec{r}-\vec{r}^{\prime}\right) e_{2}\left(\vec{r}^{\prime}\right) d \vec{r}^{\prime}$, where $U\left(\vec{r}-\vec{r}^{\prime}\right)$ is the compatibility potential. Notice that whereas the non-OP contribution is local when expressed in terms of $e_{1}$ and $e_{3}$, it becomes nonlocal when expressed in terms of $e_{2}$. This is precisely the reason that local perturbations have large-scale effects. The potential $U(\vec{r}-$ $\vec{r}^{\prime}$ ) is anisotropic and decays as $1 / r^{2}$ providing the longrange interaction between transformed regions. This can be seen more easily in $k$ space [20],

$$
U\left(k_{x}, k_{y}\right)=A_{3} \frac{\left(k_{x}^{2}-k_{y}^{2}\right)^{2}}{\left(A_{3} / A_{1}\right) k^{4}+8\left(k_{x} k_{y}\right)^{2}},
$$

which makes it clear why crosshatched correlations along the diagonals $k_{x}= \pm k_{y}$ are favored. Such correlations are more likely and stronger for larger values of $A_{3}$ whereas, formally, they vanish when $A_{3} \rightarrow 0$. We point out that such correlations are at the origin of both tweed textures and martensitic twins.

In reduced units, the numerical values of model parameters can be expressed as $\alpha_{\eta}=0.324 \kappa / l_{0}^{2}, \beta=-276 \kappa / l_{0}^{2}$, $\gamma=4.86 \times 10^{5} \kappa / l_{0}^{2}, A_{3}=4.54 \kappa / l_{0}^{2}, A_{1}=2.27 \kappa / l_{0}^{2}$ (they are the same as used in [20] and are fixed from experimen- tal data for Fe-Pd). We set $\kappa, T_{c}$, and $\alpha_{T}$ equal to unity, leading to a length scale of $l_{0}=0.23925 \mathrm{~nm}$. Hereafter the correlation length for the disorder is held fixed to $\sigma=$ $20 l_{0}$ and we perform the present study as a function of the elastic anisotropy factor $\mathcal{A}=C_{44} / C^{\prime}=A_{3} / 2 A_{2}$ by changing the value of $A_{3}$ (at constant $T, \mathcal{A} \sim A_{3}$ ) but keeping constant the ratio $A_{3} / A_{1}=2$. We have checked that variations of this value do not modify our findings qualitatively. Simulations have been carried out on a square lattice of linear size $L=10^{3} l_{0}$, discretized on a $512 \times 512$ mesh, subjected to periodic boundary conditions. Starting from a given initial configuration, we use purely relaxational dynamics in order to obtain stabilized configurations. Averages have been carried out over a sufficiently large number of independent realizations of the disorder.

Figure 1 shows snapshots of representative configurations obtained as a function of $T$ for three different values of $A_{3}$. At the right side of each configuration we have plotted the local strain distribution averaged over 10 different realizations of the disorder. In the high-temperature phase and for the three values of $A_{3}$ the distribution is single peaked around zero strain. In spite of some differences, when decreasing $T$ this peak evolves towards a twopeak distribution corresponding to the two possible values of the OP. Nevertheless, only for the largest value of $A_{3}$ [column (I)] the configuration shows the characteristic twinned martensitic structure. This is consistent with the fact that for a given value of $T$ the anisotropy $\mathcal{A}$ decreases from left to right and the system loses directionality when decreasing the value of $A_{3}$. Interestingly, for the smallest value of $A_{3}$ [column (III)] we obtain a nanocluster phaseseparated state. Tweed textures can be observed in case (I) at intermediate temperatures above the phase transition $T_{t} \simeq 1$.

To confirm the above picture we have studied the behavior of several response functions in the above model.

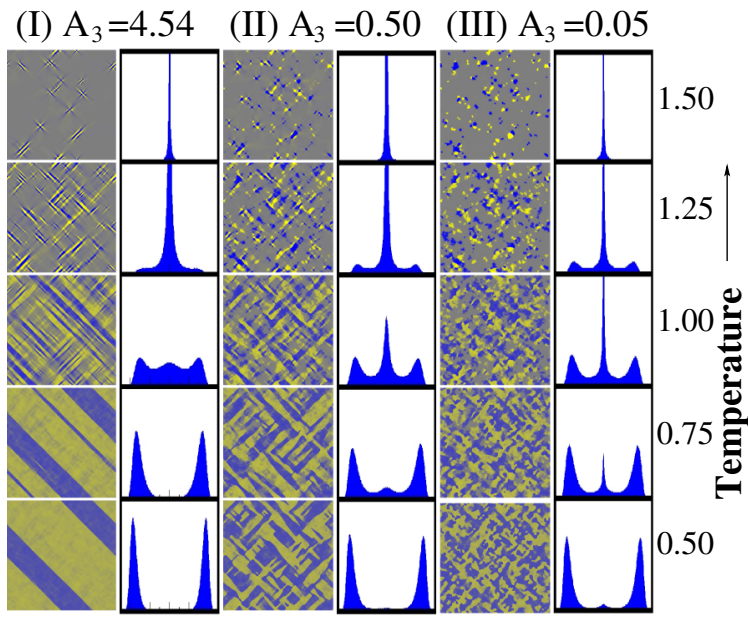

FIG. 1 (color online). Snapshots of selected configurations and local strain distribution, for three different values of $A_{3}$, as a function of $T$. The elastic anisotropy $\mathcal{A}$ decreases from left to right and the temperature from top to bottom. 


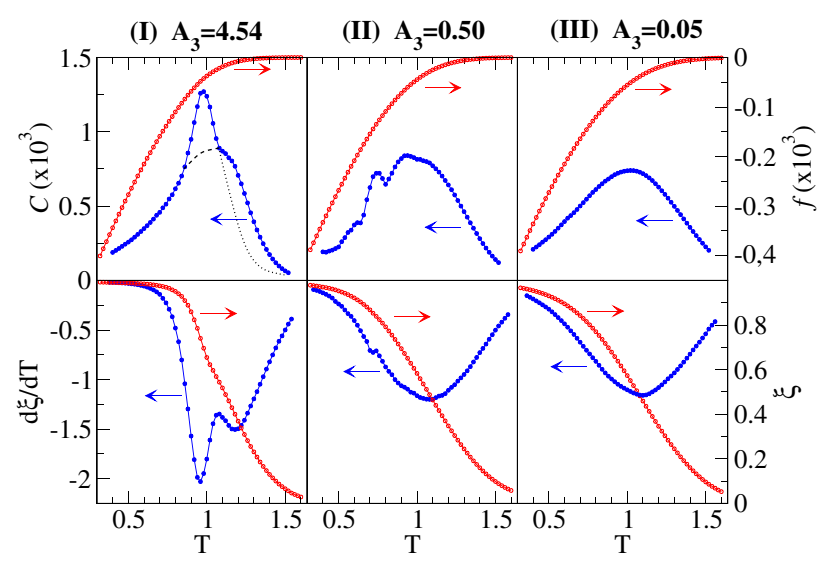

FIG. 2 (color online). Upper panels: Temperature behavior of energy density $(f)$ and heat capacity $(\mathcal{C})$ for the same three values of $A_{3}$ shown in Fig. 1. Lower panels: Temperature behavior of transformed fraction $(\xi)$ and $\xi^{\prime}=d \xi / d T$, again for the same values of $A_{3}$. In all three cases, $\mathcal{C}$ and $\xi^{\prime}$ display anomalies at the same values of $T$. Dotted lines in (I) are guides to the eyes intended to differentiate between the two underlying contributions: inhomogeneous textures and structural transition.

The upper panels of Fig. 2 show the temperature dependence of the energy density $f$ (right axis) and the heat capacity $\mathcal{C}$ (left axis) for the same three values of $A_{3}$. The behavior is compared with that obtained from the transformed fraction $\xi$ (right axis) and its first derivative $\xi^{\prime}=$ $d \xi / d T$ (left axis), displayed in the lower panels. It is worth noting that, for each value of $A_{3}$, both $\mathcal{C}$ and $\xi^{\prime}$ exhibit anomalies at the same values of $T$. For the largest value of $A_{3}$ [column (I)], $\mathcal{C}$ shows a broad bump and a sharp peak around $T \lesssim 1$. The bump is associated with the development of tweed precursors whereas the peak is a signature of the structural transition to the twinned phase (martensite). In case (II), $\mathcal{C}$ shows a smoother and lower peak that can be observed over the bump at $T \simeq 0.75$. This peak is related to the low temperature stability limit of the high-temperature phase. This is in agreement with the remaining small peak centered at zero observed in the local strain distribution at $T \simeq 0.75$ and shown in Fig. 1. In case (III) only the smooth bump associated with nanoclusters of the phase-separated pattern is observed. In any case, notice that some amount of the high-temperature phase remains down to the very low temperature ("retained austenite"; see Fig. 1).

Figure 3 shows the behavior of $\mathcal{C}$ for several values of $A_{3}$ ranging from $A_{3}=0$ to $A_{3}=10$. The observed general trends are those described above and the peak shifts to lower temperatures as $A_{3}$ decreases. This is due to the first order character of the transition and to the fact that these simulations have been performed by cooling from the hightemperature phase, in order to reproduce the standard experimental procedure. Nevertheless, we have performed a few simulations by heating the system from the lowtemperature initial configuration and found that the shifting of the peak is now towards higher temperatures thus confirming that it is related to the limit of metastability. Notice that only for values of $A_{3} \geq 1$ the peak can be associated

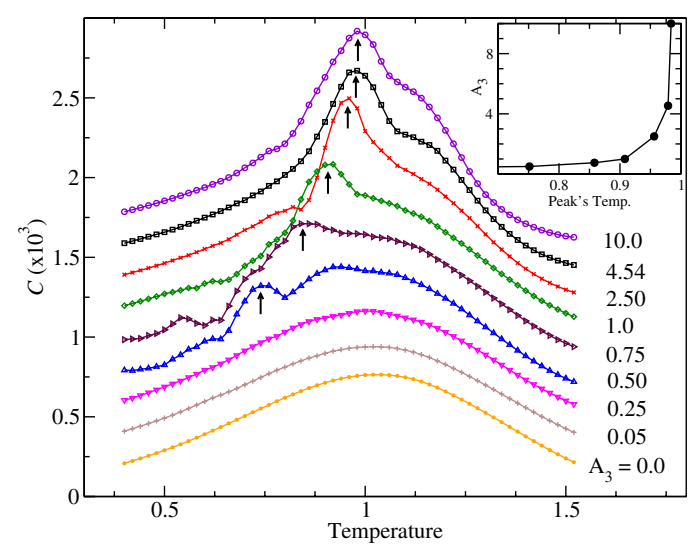

FIG. 3 (color online). Heat capacity $\mathcal{C}$ as a function of $T$ for different values of $A_{3}$. The characteristic peak shifts, diminishes, and finally disappears as $A_{3}$ is decreased. For the sake of clarity the curves are successively shifted. Arrows indicate the position of the peak for each value of $A_{3}$ and are displayed in the inset.

with the structural transition, accompanied by the development of long-range order (twinned structure). For values of $A_{3} \lesssim 1$ the peak is associated with the stability limit of the high-temperature phase and rapidly goes to zero as $A_{3} \rightarrow$ 0 . This can be seen in the inset of Fig. 3 where we have plotted the peak temperature as a function of $A_{3}$. We emphasize that comparable anomalies have been found experimentally [16] in $\mathrm{Ni}-\mathrm{Ti}(\mathrm{Fe})$ alloys, where an increase in $\mathrm{Fe}$ content diminishes and shifts the peak in $\mathcal{C}$ to lower temperatures. In particular, it is found that for 6 at. $\% \mathrm{Fe}$, no signature of peak in $\mathcal{C}$ is observed and the transition is suppressed.

Figure 4 shows ZFC simulations for different values of $A_{3}$. For $A_{3}=0.05$ [case (III)] the curve represents an elastic behavior that deviates from the FC curve below the peak temperature (shown in the upper inset). This is in very good agreement with experimental results in

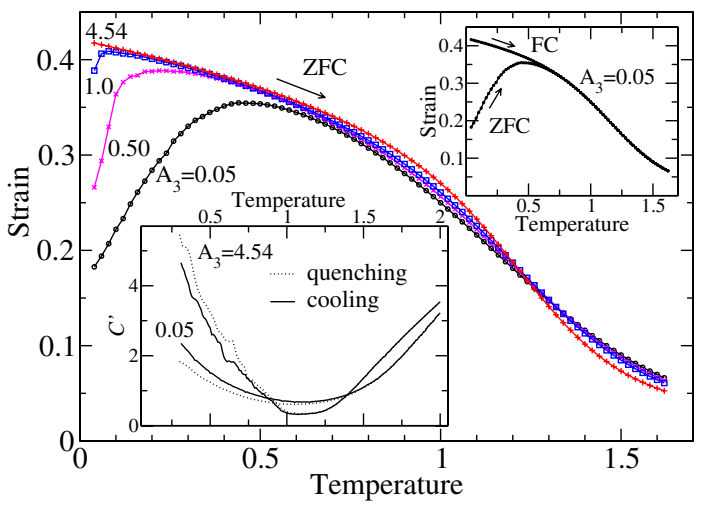

FIG. 4 (color online). The ZFC curves for different values of elastic anisotropy $A_{3}$. ZFC and FC curves for very low anisotropy $\left(A_{3}=0.05\right)$ shown in the upper inset exhibit a strain glass behavior for the nanoscale phase-separated state. The lower inset contrasts the elastic response $C^{\prime}$ of the system to a constant external stress field for two very different values of $A_{3}$. For details, see the text. 
$\mathrm{Ni}_{51.5} \mathrm{Ti}_{48.5}$ that corroborate the suggested glassy behavior [18]. As expected, our simulations show that this behavior progressively disappears for increasing values of $A_{3}$. This trend indeed corresponds to an increase in the elastic anisotropy $\mathcal{A}$ and favors the development of long-range order. The lower inset shows the elastic response $C^{\prime}$ of the system to an external stress field for $A_{3}=4.54$ [case (I)] and $A_{3}=0.05$ [case (III)]. For each of these values, cooling and quenching curves have been calculated independently, giving rise to very similar behavior, indicating the robustness of the behavior. For $A_{3}=4.54$, the pronounced dip of the curve clearly confirms the existence of a phase transition. In addition, similar to the behavior observed in the heat capacity curves, a flat region lies in the temperature range $1.0 \leqq T \lesssim 1.25$ that coincides with the precursor (tweed) region. Instead, for $A_{3}=0.05$, the dip amplitude becomes much lower so that the curve and thus the transition broadens. Similar qualitative results have been obtained experimentally in the elastic response when varying the composition in Ni-rich $\mathrm{Ni}_{50+x} \mathrm{Ti}_{50-x}$ alloys [17].

In summary, disorder is important since the main results are a consequence of a competition or cooperation between two factors: the long-range anisotropic interactions [mediated by $U(\vec{k})$ in Eq. (1)] and the quenched-in disorder. For large values of the elastic anisotropy $\mathcal{A}$ tweed is obtained above the transition temperature $T_{t}$ as a cooperative phenomenon between the two. Instead, below $T_{t}, U(\vec{k})$ provides the system with an easy channel to optimize the total free energy by means of inducing strain correlations along the diagonals and thus giving rise to twin boundaries: this is a clear triumph of anisotropy over disorder. Nevertheless, for low values of $\mathcal{A}$ the potential $U(\vec{k})$ is weak and thus fails the system to achieve its twinned state. Therefore, the only way for the strain to stabilize is to create domains ("ramified droplets") that accommodate it according to the disorder of the system. Thus, the specific patterns obtained can depend on the characteristics of the disorder. In fact, in this case the existence of two martensitic variants $\left(e_{2}= \pm e\right)$ with the same energy implies the existence of a large number of configurations of similar energy. Only the gradient term favors the growth of the domain size, but mostly the energy barriers at the boundaries of different variants are too high for the system to overcome. At this point, frustration arises as a consequence of the disorder, so that the system anchors itself in a frozen metastable state. Eventually, dynamics could become extremely slow, resulting in a glassy behavior as reported by Sarkar et al. [17]. These features are also reflected in the calculated response functions. The behavior of the heat capacity $\mathcal{C}$ clearly highlights the main results of our model. The shift in the peak can be understood in terms of the potential energy. According to Eq. (1) the long-range anisotropic potential $U(\vec{k})$ is a nonlocal extra contribution to the harmonic term. Thus, it has an influence on $T_{c}$ : a drop in the weight of this term would imply a drop in the effective $T_{c}$, as shown in the inset of Fig. 3. Finally, for very low anisotropy we obtained a glassy behavior consistent with recent $\mathrm{ZFC}$ and $\mathrm{FC}$ experiments [18].

Note that in a broad class of functional materials (including high-temperature superconductors and colossal magnetoresistance manganites [1,2], ferromagnets $[7,8]$ and ferroelectrics [9]) a clear dependence of their properties on the intrinsic inhomogeneities has been found, and where strain and disorder play a key role. Sometimes these inhomogeneities even give rise to entirely new properties, absent in pure materials. In line with this general observation, both the SMAs Fe-Pd and Ni-Ti considered here drastically change their behavior when the composition is varied slightly $[17,20]$. A rich behavior landscape, tweed, suppression of the martensitic transformation, emergence of new structures and glassy behavior, has been found, which has been observed in experiments and is a signature or consequence of such ferroic inhomogeneities.

This work was supported by CICyT (Spain) Project No. MAT2004-01291, DURSI Catalonia) Project No. 2005SGR00969, Marie-Curie RTN MULTIMAT (EU), Contract No. MRTN-CT-2004-505226, and the U.S. Department of Energy. P. Ll. acknowledges support from DGICyT (Spain).

[1] E. Dagotto et al., Science 309, 257 (2005).

[2] A. R. Bishop et al., Europhys. Lett. 63, 289 (2003).

[3] T. Castán et al., in Magnetism and Structure in Functional Materials, edited by A. Planes, Ll. Mañosa, and A. Saxena (Springer, Berlin, 2005), pp. 27-48, and references therein.

[4] L. E. Tanner et al., J. Phys. (Paris), Colloq. 43, C4-169 (1982).

[5] Although the precise periodicity in the tweed structure depends critically on the imaging conditions, there exists a general agreement in that it is on the scale of ten to hundreds of lattice constants.

[6] A. Saxena et al., Phys. Rev. Lett. 92, 197203 (2004).

[7] Y. Murakami et al., Acta Mater. 50, 2173 (2002).

[8] M. Porta et al., Phys. Rev. B 72, 054111 (2005).

[9] N. Mathur and P. Littlewood, Nat. Mater. 3, 207 (2004).

[10] Y. Imry and M. Wortis, Phys. Rev. B 19, 3580 (1979).

[11] A. Planes and Ll. Mañosa, Solid State Phys. 55, 159 (2001), and references therein.

[12] K. Otsuka and X. Ren, Prog. Mater. Sci. 50, 511 (2005).

[13] Y. Murakami et al., J. Microsc. 203, 22 (2001).

[14] S. M. Shapiro et al., Phys. Rev. Lett. 57, 3199 (1986).

[15] R. Oshima et al., Metall. Trans. A 19, 803 (1988).

[16] M.-S. Choi et al., Scr. Mater. 53, 869 (2005).

[17] S. Sarkar et al., Phys. Rev. Lett. 95, 205702 (2005).

[18] Y. Wang et al., Phys. Rev. B 76, 132201 (2007).

[19] S. Kartha et al., Phys. Rev. Lett. 67, 3630 (1991).

[20] S. Kartha et al., Phys. Rev. B 52, 803 (1995).

[21] S. R. Shenoy et al., Phys. Rev. B 60, R12 537 (1999).

[22] A. Saxena and T. Lookman, in Handbook of Materials Modeling, edited by S. Yip (Springer, Berlin, 2005), pp. 2143-2155. 